\title{
Aminoguanidine hydrazones (AGH's) as modulators of norfloxacin resistance in Staphylococcus aureus that overexpress NorA efflux pump
}

\author{
Natalina Dantas ${ }^{\mathrm{a}}$, Thiago Mendonça de Aquino ${ }^{\mathrm{b}}$, João Xavier de Araújo-Júnior ${ }^{\mathrm{b}}$, \\ Edeildo da Silva-Júnior ${ }^{\mathrm{b}}$, Ednaldo Almeida Gomes ${ }^{\mathrm{b}}$, Antoniel Augusto Severo Gomes ${ }^{\mathrm{c}}$, \\ José Pinto Siqueira-Júnior ${ }^{\mathrm{a}, * *}$, Francisco Jaime Bezerra Mendonça Junior ${ }^{\mathrm{d} \text {,* }}$ \\ a Laboratório de Genética de Microorganismo, Departamento de Biologia Molecular/CCEN/Universidade Federal da Paraíba-UFPB, 58051-970 João Pessoa, PB, Brazil \\ ${ }^{\mathrm{b}}$ Laboratório de Química Medicinal, Escola de Enfermagem e Farmácia, Universidade Federal de Alagoas/UFAL, 57072-970 Macéio, AL, Brazil \\ ${ }^{\mathrm{c}}$ Departamento de Física e Biofísica, Instituto de Biociências, Universidade Estadual Paulista, UNESP, 18618-000 Botucatu, SP, Brazil \\ d Laboratório de Síntese e Planejamento de Fármacos, Departamento de Ciências Biológicas/CCBSA, Universidade Estadual da Paraíba-UEPB, 58070-450 João Pessoa, \\ PB, Brazil
}

\section{A R T I C L E I N F O}

\section{Keywords:}

Staphylococcus aureus

Efflux pump inhibitors

Docking

Aminoguanidine hydrazones

\begin{abstract}
A B S T R A C T
One of the promising fields for improving the effectiveness of antimicrobial agents is their combination with efflux pump inhibitors (EPIs), which besides expanding the use of existing antibiotics. The goal of this research was to evaluate a series of aminoguanidine hydrazones (AGH's, 1-19) as antibacterial agents and NorA efflux pump inhibitors in Staphylococcus aureus strain SA-1199B. Molecular modeling and docking studies were also performed in order to explain at the molecular level the interactions of the compounds with the generated NorA efflux pump model. The MICs of the antibiotic and ethidium bromide were determined by microdilution assay in absence or presence of a subinhibitory concentration of aminoguanidine hydrazones and macrophages viability was determined through MTT assay. Bioinformatic software Swiss-Model and AutoDock 4.2 were used to perform modeling and docking studies, respectively. As results, all AGH's were able to potentiate the action for the antibiotic norfloxacin, causing MIC's reduction of 16-fold and 32-fold to ethidium bromide. In the cell viability test, the concentration of $10 \mu \mathrm{g} / \mathrm{mL}$ showed better results than $90 \%$ and the concentration of $1000 \mu \mathrm{g} / \mathrm{mL}$ showed the lowest viability, reaching a maximum of $50 \%$ for the analyzed aminoguanidine hydrazones. Molecular docking studies showed that both norfloxacin and derivative $\mathbf{1 3}$ were recognized by the same binding site of NorA pump, suggesting a competitive mechanism. The present work demonstrated for the first time that AGH derivatives have potential to be putative inhibitors of NorA efflux pump, showing a promising activity as an antibacterial drug development.
\end{abstract}

\section{Introduction}

The emergence of drug resistance in different species of bacteria is a growing cause of concern [1]. These bacteria include strains of Staphylococcus aureus, which is an opportunistic but potentially serious human pathogen that can be resistant to vancomycin [2], and also to the recently discovered linezolid [3], which is often considered "the last line of defense" against multidrug resistance (MDR) S. aureus strains.

Among the MDR efflux pumps present in this species, NorA, which belongs to the Major Facilitator Superfamily (MFS) is considered representative, and the most efficient of the MDR systems in Grampositive bacteria. In particular, the NorA protein confers resistance to a wide range of structurally unrelated antibiotics and antiseptics such as acridines, ethidium bromide, pentamidine, or more importantly, the hydrophilic fluoroquinolones, an important class of broad-spectrum antimicrobials with potent activity [4-6].

The structural biology data of NorA are still undetermined. However, through sequence homology studies and the sharing of various substrates with other MDR pumps, the hypothesis that NorA may have a large hydrophobic binding site [7] has gained acceptance. This

\footnotetext{
Abbreviations: EPIs, efflux pump inhibitors; AGH's, aminoguanidine hydrazones; MICs, minimum inhibitory concentrations; MTT, 1-(4,5-dimethylthiazol-2-yl)-3,5-diphenyl formazan;

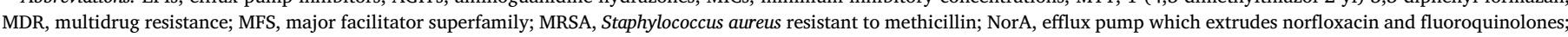

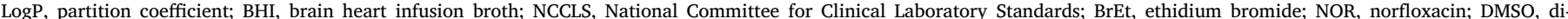
methylsulfoxide; DMEM, Dulbecco's Modified Eagle Medium; FBS, foetal bovine serum; PDB, protein database bank; PAßN, dipeptide amide

* Corresponding author.

*** Corresponding author.

E-mail addresses: jpsiq@uol.coml.br (J.P. Siqueira-Júnior), franciscojbmendonca@yahoo.com.br (F.J.B. Mendonça Junior).
} 
peculiarity could explain MDR pumps' broad specificity for substrates.

Even though new methods of treatment have been developed, or are about to be made available, the restoration of clinical antibacterial efficacy (against which resistance has developed), remains an important goal. One of the promising fields for improving the effectiveness of antimicrobial agents is combining them with efflux pump inhibitors (EPIs). This expands the usefulness of existing antibiotics and reduces the emergence of resistant mutant strains [8].

Efforts are underway to generate new antibacterial agents able of circumventing drug efflux by synthesizing compounds that are poor pump substrates, or to identify compounds that reduce or block efflux pump activity [9].

Among these compounds, the aminoguanidine hydrazones (AGH) derivatives may well become a source for new candidates to develop alternatives to antibiotics, and to combat bacterial resistance. AGH represent a class of compounds containing an amidine group (guanyl), connected to a hydrazone moiety [10]. Along with the biological potency of these molecules come; antihypertensive [11,12], antidiabetic [13], antineoplastic [14,15], antitubercular [16], antimalarial, antiLeishmania, antifungal, antibacterial, and anti-HIV activities [17-19].

In view of these initial considerations, and in order to explore the antibiotic potential of AGHs, which may lead to the synthesis of new structurally related derivatives, the goal of this research was to evaluate a series of AGHs as antibacterial agents and NorA efflux pump inhibitors in Staphylococcus aureus resistant to methicillin (MRSA), and their cytotoxicity on macrophages. Molecular modeling and docking studies were also performed and proved necessary in order to explain at the molecular level the interactions of the compounds with the generated NorA efflux pump model.

\section{Experimental}

\subsection{Chemistry}

The AGH (1-19) involved in this study were previously synthesized by reaction of the appropriate aldehyde with aminoguanidine hydrochloride in refluxing $95 \%$ ethanol. The title compounds were isolated after cooling to room temperature, being washed, or recrystallized using an appropriate solvent. Structural characterization of the products is described elsewhere $[20,21]$. LogP was calculated in the HyperChem $^{\mathrm{TM}}$ software Release 8.0.8 Windows.

\subsection{Biological assays}

\subsubsection{Bacterial strains}

The $S$. aureus strain used, SA-1199B, over expresses the NorA gene encoding the NorA efflux protein [22], which extrudes hydrophilic fluoroquinolones and other drugs such as DNA-intercalating dyes. The strain, kindly provided by Professor Simon Gibbons (University of London), was maintained on blood agar base slants (Laboratory Difco Ltda., Brazil). Prior to use, the cells were grown overnight at $37^{\circ} \mathrm{C}$ in brain heart infusion broth (BHI-Laboratory Difco Ltda., Brazil).

\subsubsection{Antibiotics and nucleic-acid binding (NAB) compounds}

All antibiotics were prepared according to NCCLS guidelines [23], and were purchased from Sigma-Aldrich Co. The stock solution of ethidium bromide (BrEt), norfloxacin (NOR) and perfloxacin were prepared in distilled water. The stock solutions of the title compounds were prepared in DMSO solutions where, at its highest final concentration after dilution in broth (4\%), no bacterial growth inhibition occurred [22].

\subsubsection{Susceptibility tests}

The minimum inhibitory concentrations (MICs) of the antibiotics, BrEt and AGH derivatives were determined by microdilution assay using a suspension of ca. $10^{5} \mathrm{cfu} / \mathrm{mL}$ and drug concentrations in the range of $256-0,25 \mu \mathrm{g} / \mathrm{mL}$ (two-fold serial dilutions). MIC is defined as the lowest concentration at which no growth is observed. AGH compounds were evaluated as NorA efflux pump inhibitors based on their effects on MICs of the antibiotic and ethidium bromide, in the presence and absence of AGH's at sub inhibitory concentrations and corresponding to $1 / 4$ of their own MICs [24]. All experiments were carried out at least twice with consistent results.

\subsubsection{In vitro cytotoxicity on macrophages}

Macrophages viability was determined thought the MTT 1-(4,5-dimethylthiazol-2-yl)-3,5-diphenyl formazan colorimetric assay [25]. Approximately $1,5 \times 10^{5}$ macrophages (line J774) per well were seeded into 96-well plate plates and were cultured in high glucose Dulbecco's Modified Eagle Medium (DMEM: Life Technologies cat \# 11995073) supplemented with $10 \%$ foetal bovine serum (FBS: Life Technologies cat \# 16000044) in the presence or absence of aminoguanidine hydrazone derivatives at $37{ }^{\circ} \mathrm{C}$ and $5 \% \mathrm{CO}_{2}$ for $24 \mathrm{~h}$ in three different concentrations $(10,100$ e $1000 \mu \mathrm{g} / \mathrm{mL})$. One hour before addition of MTT, $2 \mu \mathrm{L}$ of Triton 100X were added to 3 wells for cell death comparison. After incubation, cytotoxicity was determined by adding $100 \mu \mathrm{L}$ of MTT $(500 \mu \mathrm{g} / \mathrm{mL})$ to each well and further incubated for $2 \mathrm{~h}$. The supernatant was discarded and the precipitate was resuspended in $100 \mu \mathrm{L}$ of DMSO. Finally, readings were performed at $540 \mathrm{~nm}$ on a microplate reader (BioTek Instruments, Inc., Winooski, VT, USA). Optical density data (cell viability) were obtained in quadruplicate and presented as mean \pm standard deviation. The MTT reduction activity was determined as \% cell viability, calculated by the formula: ([absorbance of treated cells/absorbance of untreated cells] x100). Data were analyzed using Origin (Version 6.1052; Origin Lab Corp Northampton, MA, USA). One-way ANOVA followed by Tukey test was used to compare differences between groups, and data were considered statistically significant for $p$ value $\leq 0,05$.

\subsection{Molecular modeling studies}

\subsubsection{NorA modeling}

In order to study the structural determinants of the NOR site, it was used $S$. aureus NorA pump primary sequence, taken from the Reference Sequence NP_373905.1 and SWISS-MODEL software [26] to produce a structural model of the protein. The monomer A of the crystal structure of YajR transporter from E. coli (PDB id: 3WDO) [27] was selected as template for molecular modeling, according to the HMM-HMM-based lightning-fast iterative sequence search (HHBlits) tool which uses the database SWISS-MODEL (SMTL) from the selected sequence. Best alignments were classified according to the sensitive HMM-HMM-based lightning-fast iterative sequence search (HHBlits) tool parameters. NorA model was constructed with ProMod v.3.7 program [28].

\subsubsection{Docking}

In order to find structural insights, best modifying activity antibiotic in SA-1199B strains compound 13 (having better antibiotic modifying activity in the SA 1199B strain) and NOR were used to perform Molecular Docking on the generated model of NorA efflux pump using Autodock 4.2 [29]. The structures of both molecules were build using the server PRODGR [30]. Receptor preparation was carried out using model previously generated by SWISS-MODEL [26] software where was add hydrogen atoms and Gasteiger charge to entire molecule. Autogrid was carried out for the preparation of the grid map, where a grid box was 40-40-40 $\AA$ npts, spacing was $0.603 \AA$ and grid center was $-29.984,55.831$ e 73.869 to $x, y$, and z-axis. Ligand preparation was set out with flexible bonds at maximum to both compounds. Further, Autodock (protein-rigid and ligand-flexible) were performed using Genetic Algorithm (GA) in standard configuration, with a number of GA runs and a maximum number of individuals survive at 20 and 2, respectively. Best pose of each docking calculation was selected and analyzed using PyMol software [31]. 


\section{Results and discussion}

\subsection{Antibiotic susceptibility and cytotoxicity}

In this study, in vitro, and in silico assays were employed for searching new antibacterial agents that can also act as putative inhibitors of efflux pumps [32], being, therefore, more effective as antibiotic drugs due to their dual profile that hinders or slows the development of resistant strains.

Among the evaluated compounds, only AGH's 10, 14 and 17 (MIC $=16,16,32 \mu \mathrm{g} / \mathrm{mL}$, respectively) showed higher antibacterial activity than the standard antibiotic NOR (MIC $=128 \mu \mathrm{g} / \mathrm{mL}$ ), being 4 to 8 times more active. All other AGH's did not display relevant antibacterial activity at the evaluated concentrations, showing MICs ranging from 64 to $>256 \mu \mathrm{g} / \mathrm{mL}$.

The antibacterial activity observed in compounds 10, 14 and 17, may be partly associated to the hydrophobic characteristic of these derivatives. Compounds 10 (with 3,5-di-tert-butyl radical) and 14 (with 4-phenyl radical) have the highest LogP values of the series: 2.37 and 1.37, respectively. Exception is compound 17 with 3,4-dichloro substitution, which presents $\log \mathrm{P}=0.32$ and is also active, but two times less (MIC $=32 \mu \mathrm{g} / \mathrm{mL}$ ).

The size of the side chains, as well as substitutions on the guanidine nucleus both, also seem to play key roles in the antibacterial activity [33], probably acting as membrane lipid affinity modulators [32]. Previously studies of the staphylococcal activity of $\mathrm{N}$-acylhydrazones also describe the relationship between the decrease of the antibiotic activity and the reduction of the lipophilicity of the compounds [18].

Unlike observed with their antibacterial activity, where few compounds proved to be active (but better than NOR), the evaluated compounds presented a potent efflux pump inhibitory activity when were added to the growth medium at sub-inhibitory concentrations in association with the antibiotics (NOR, BrEt and Perfloxacin) in a serial dilution.

We observed that the corresponding MIC was reduced by at least two-fold, (and up to 16-fold and 32-fold for NOR and BrEt, respectively). Compounds $3, \mathbf{1 3}$, and 17 are the most active compounds and provided the lowest MIC's values ( $8 \mu \mathrm{g} / \mathrm{mL}$ ), followed by compounds 2 , $8,9,12,16,18$ and $19(16 \mu \mathrm{g} / \mathrm{mL})$, reducing NOR MIC by 16 -fold and 8 -fold, respectively and BrEt MIC in up to 32 times The AGH derivatives did not change the MIC value of perfloxacin (negative control for efflux pump) (MIC $=16 \mu \mathrm{g} / \mathrm{mL}$ ) when incubated in the association. (Table 1 ).

This potent activity can be partially explained by the presence of electron withdrawing substituents such as nitrile (compound 3), a quinolone (compound 13), ester (compound 16), chlorine (compounds 12, 17 and 18) and bromine (compound 8) in the phenyl ring. These groups have been noted already in the literature as playing a key role in other structures such as piperine and omeprazole analogs with potent NorA inhibitory activity [34-36].

Substitution by electron donors groups as di-tert-butyl (compound 10), hydroxyl and methoxyl (compounds 4, 5, 6 and 7) are shown to be unfavorable for the modulatory activity providing the less active compounds of the series (MIC $=64 \mu \mathrm{g} / \mathrm{mL}$ - NOR reductions $=2$-fold), being less actives than the unsubstituted prototype, compound 15 with MIC $=32 \mu \mathrm{g} / \mathrm{mL}$ NOR reductions $=4$-fold.

The only unable compound to modulate the activity of NOR was the zwitterion 11, which has a carboxylic acid group linked to the C-4 position. We believe that their inactivity may have been associated from its low solubility in the culture medium.

It is likely that the guanidine moiety also plays a vital role in their antibiotic adjuvant activity, as observed in other studies concerning the antimicrobial properties of AGH's [37,38]. As an example, several antimicrobial peptides polycationic rich in arginine indicates the importance of the cation portion (positive charge) of guanidine group for its antimicrobial properties [38]. This portion is linked to the molecular recognition process of proteins and enzymes, which by this function is performed electrostatic interactions and hydrogen bonds [39].

In order to evaluate the cytotoxic profile of the evaluated compounds, cytotoxicity assay in macrophages (line J774) were performed in three concentrations $(10,100$ e $1000 \mu \mathrm{g} / \mathrm{mL})$.

According to the cell viability (Graph 1), at $10 \mu \mathrm{g} / \mathrm{mL}$ all compounds, except 4, 8, 14 and 17 showed cellular viability high than $90 \%$. At $100 \mu \mathrm{g} / \mathrm{mL}$, half of the compounds $(1,3,4,5,6,7,9,10,11$ and 16) showed cell viability high than $80 \%$. Compounds 18 and 19 showed cell viability around $50 \%$, and seven compounds $(2,8,12,13,14,15$ and 17) showed cell viability lower than $20 \%$ and were considered toxic. At $1000 \mu \mathrm{g} / \mathrm{mL}$ all compounds showed a low cell viability less than $20 \%$, except for 4, 10 and 11, which showed cell viability above $50 \%$.

Since the compounds were evaluated for their modulatory activity at sub inhibitory concentrations, corresponding to $1 / 4$ of their own MICs $4 \mu \mathrm{g} / \mathrm{mL}$ (for 10 and 14); $8 \mu \mathrm{g} / \mathrm{mL}$ (for 17), and $64 \mu \mathrm{g} / \mathrm{mL}$ (for all other compounds), the cytotoxicity evaluation indicate that all the compounds showed lower or no-toxicity for macrophages in the evaluated concentrations.

\subsection{Molecular modeling and docking studies}

Several efflux pump inhibitors (EPIs) are currently under study, but the elucidation of their mechanisms of action remain largely unknown [40]. Therefore, knowing that NorA efflux pump is a membrane protein and its structure was not revealed until then, the proposition of theoretical models using bioinformatics as research tool becomes a good alternative to carrying out molecular interaction studies and to identify binding sites for potential ligands.

The search for the NorA protein model by the SWISS-MODEL resulted in the YajR model of the Major Transporter Superfamily (MFS), E. coli (PDB id: 3WDO) [27], which was used to construct the theoretical model of NorA protein (Fig. 1).

The results for QMEAN4, GMQE, sequence identity, sequence similarity and coverage were $-11.00,0.51,16.03,0.29$ e 0.95 , respectively.

These generated three-dimensional model of NorA presented satisfactory values of identity (16.03) and similarity sequence (0.29), presenting a high coverage and similarity of $95 \%$ of the structure, with preservation of the secondary structure varying only the constituent amino acid residues of the NorA protein (Fig. 1). These values were similar to the model found by Kalia et al. [41], for the glycerol-3phosphate transporter model.

Another interesting finding was that QMEAN4 value is highly negative, which corresponds to an unfavorable solvation potential, typically observed in transmembrane proteins due to their outward hydrophobic residues [42].

Superficially, as expected, the central outer portion of both structures showed a high density of hydrophobic residues, suggesting its transmembrane location, while their ends showed high charge density: the periplasmic portion of the generated model showed higher ion density. Since the cytoplasmic portion due to the fewer number of amino acid residues showed no C-terminal domain (Fig. 1).

Despite equivalent secondary and tertiary structures, notable surface differences were found between the crystallographic structures of the 3WDO matrix protein [27] and the generated model. These differences are due to the chemical differences particular of each protein.

Considering the functionality of both proteins, substitutions in the intermolecular channel region constitute important differences for the types of molecules transported, thus, substitutions of amino acid residues F21, M25, M121, H225, F144, M340 and Q347 of structure 3WDO By residues I12, F16, I19, T113, S215, I136, G329 and T336 of the NorA model, respectively, caused the generated model to have higher spatial volume in the intermolecular canal.

It is worth noting that the exposure of the side chain of residue I15 in the model, substituted by R24 in 3WDO, which beyond the increasing volume channel, yet allows possible interactions with certain 
Table 1

Chemical structures and LogP of AGH's and MICs of antibiotics and BrEt against S. aureus strain SA-1199B, in the presence and in absence (alone) of AGH derivatives.

\begin{tabular}{|c|c|c|c|c|c|}
\hline \multirow[t]{2}{*}{ Compounds } & \multirow[t]{2}{*}{ Aminoguanidine hydrazones } & \multicolumn{4}{|c|}{$\operatorname{MIC}(\mu \mathrm{g} / \mathrm{mL})$} \\
\hline & & $\log P$ & Norfloxacin & BrEt & Perfloxacin \\
\hline Alone & - & - & 128 & 32 & 16 \\
\hline+1 & & -1.07 & $32(4 x)^{a}$ & $8(4 x)^{a}$ & 16 \\
\hline+2 & & 0.54 & $16(8 x)^{a}$ & $4(8 x)^{a}$ & 16 \\
\hline+3 & & 0.49 & $8(16 x)^{a}$ & $1(32 x)^{a}$ & 16 \\
\hline+4 & & -1.25 & $64(\mathrm{x} 2)^{\mathrm{a}}$ & $16(\mathrm{x} 2)^{\mathrm{a}}$ & 16 \\
\hline+5 & & -1.22 & $64(\mathrm{x} 2)^{\mathrm{a}}$ & $16(\mathrm{x} 2)^{\mathrm{a}}$ & 16 \\
\hline+6 & & -1.25 & $64(\mathrm{x} 2)^{\mathrm{a}}$ & $16(\mathrm{x} 2)^{\mathrm{a}}$ & 16 \\
\hline+7 & & -0.26 & $64(\mathrm{x} 2)^{\mathrm{a}}$ & $16(\mathrm{x} 2)^{\mathrm{a}}$ & 16 \\
\hline+8 & & 0.82 & $16(\mathrm{x} 8)^{\mathrm{a}}$ & $4(x 8)^{a}$ & 16 \\
\hline+9 & & -1.03 & $16(x 8)^{\mathrm{a}}$ & $4(\mathrm{x} 8)^{\mathrm{a}}$ & 16 \\
\hline+10 & & 2.37 & $64(\mathrm{x} 2)^{\mathrm{a}}$ & 32 & 16 \\
\hline+11 & & 0.15 & 128 & 32 & 16 \\
\hline+12 & & 0.32 & $16(\mathrm{x} 8)^{\mathrm{a}}$ & $4(x 8)^{a}$ & 16 \\
\hline+13 & & -0.42 & $8(x 16)^{\mathrm{a}}$ & $1(\mathrm{x} 32)^{\mathrm{a}}$ & 16 \\
\hline+14 & & 1.37 & $32(x 4)^{a}$ & $8(x 4)^{a}$ & 16 \\
\hline+15 & & 0.77 & $32(\mathrm{x} 4)^{\mathrm{a}}$ & $8(x 4)^{a}$ & 16 \\
\hline+16 & & 0.18 & $16(x 8)^{\mathrm{a}}$ & $2(x 16)^{a}$ & 16 \\
\hline+17 & & 0.32 & $8(x 16)^{\mathrm{a}}$ & $4(x 8)^{a}$ & 16 \\
\hline+18 & & 0.54 & $16(\mathrm{x} 8)^{\mathrm{a}}$ & $2(\mathrm{x} 16)^{\mathrm{a}}$ & 16 \\
\hline+19 & & 1.37 & $16(x 8)^{\mathrm{a}}$ & $4(x 8)^{a}$ & 16 \\
\hline
\end{tabular}

${ }^{\mathrm{a}}$ Fold reduction in MIC.

elongated portions of potential ligands (Fig. 2). The polarity of the channels, both showed mostly hydrophobic residues, unless the residues E222, D307, and R310 for NorA model and H215, E318 and Q347 for 3WDO.
According to Jiang et al. [27] the mechanism of operation of the MFS efflux pumps that alternates between the inward and outward conformations, and conserves the central portion during the transportation of its specific solutes, opening for both sides. The more external 

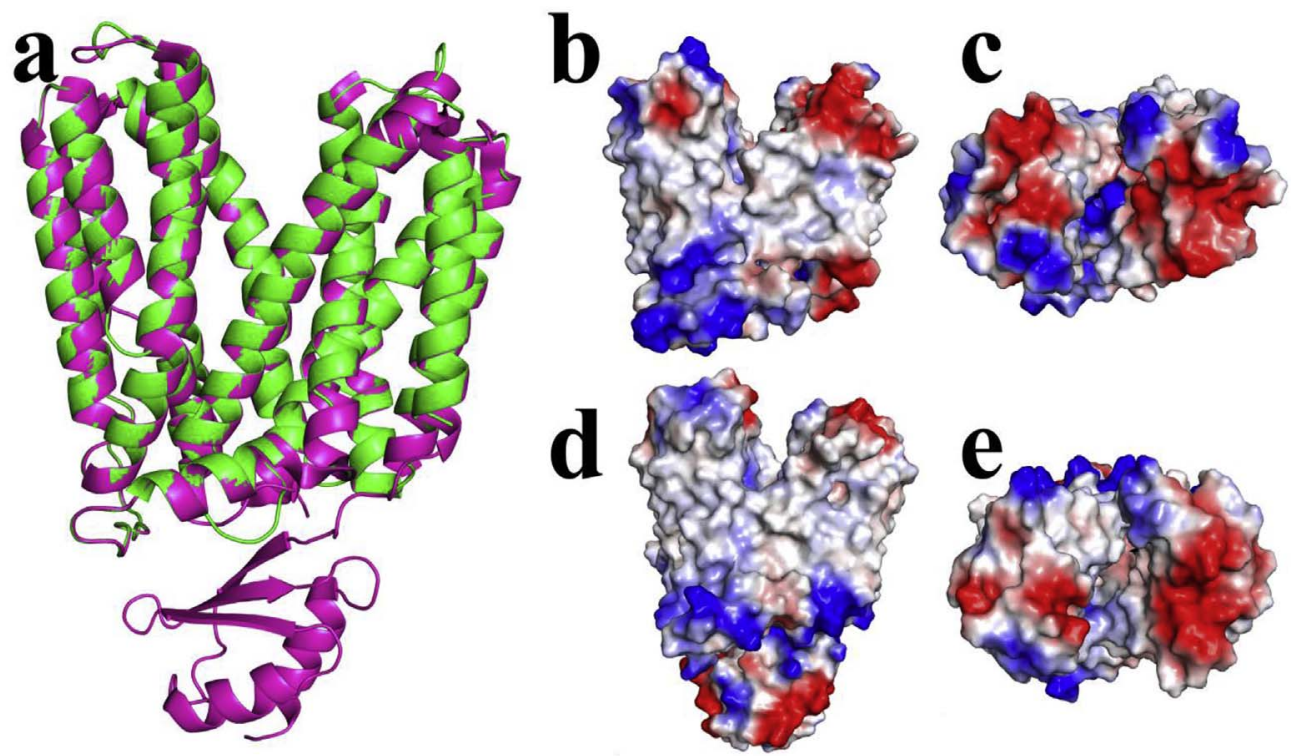

Fig. 1. Three-dimensional presentation of the crystal structure 3WDO (purple) superimposed the generated NorA model (green). Representation of the secondary structure (a); the electrostatic surface of 3WDO (b, c); the electrostatic surface of the generated NorA model (d, e) cationic residues (blue), anionic (red), and hydrophobic (white). (For interpretation of the references to color in this figure legend, the reader is referred to the Web version of this article.)

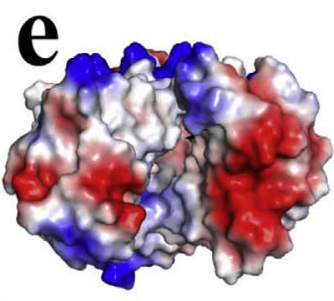

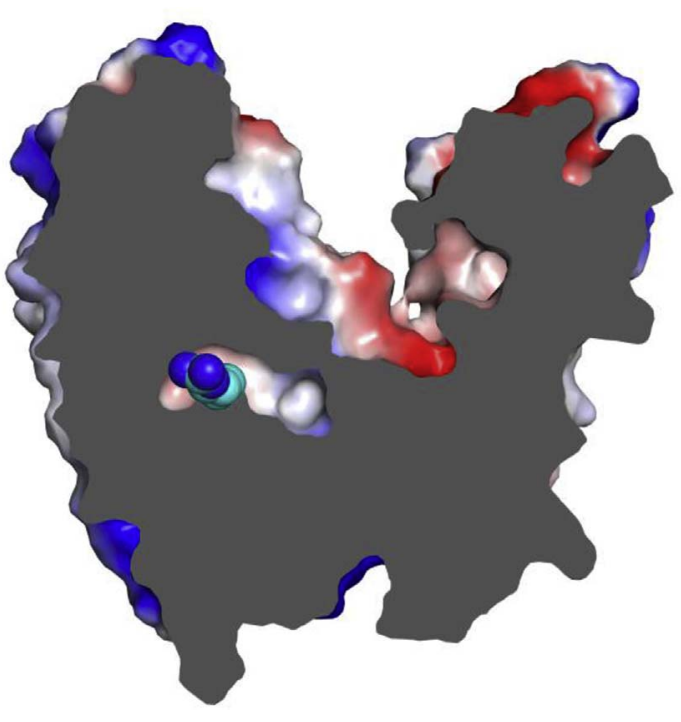

Fig. 2. Three-dimensional representations of 3WDO NorA model. The vertical cut of the electrostatic surface of the model detailing its central cavity, diminished in the structure of the 3WDO thanks to the side chain of residue R24 blue spheres in the center of the figure. Cationic, anionic and hydrophobic residues represented in blue, red and white, respectively. (For interpretation of the references to color in this figure legend, the reader is referred to the Web version of this article.)

positioning of the site found in the 3WDO structure is shown as an unlikely site for medium-sized molecules. However, such findings reinforce their specificity for small molecules, such as their natural substrates: lactose and galactose [27]. On the other hand, these same sites for NorA were located in the central region of the protein and such interaction represents a greater approximation of the models proposed until then.

In molecular docking studies, both ligands (NOR and 13) were localized in the same site: center position in the N-lobe (Fig. 3a).

The best pose docking of NOR showed an $\mathrm{E}_{\text {bind }}=-6.99 \mathrm{kcal} / \mathrm{mol}$, and it is stabilized by several amino acid residues (Fig. 3b). Hydrophobic interactions of quinolone and piperazine moieties occur with the side chains of the residues I15, F16, I19, M109 and I136. Polar interactions occur between its acidic portion and the residues N340 (2.86 and $3.28 \AA$ ) and R310 (2.88, 3.19 and $3.30 \AA$ ), and its nitrogen-para of the piperazine ring was stabilized by residue Q51 (3.07 $\AA$ ) and oxygen of the main chain of residue M107 (3.32 $\AA$ ) of the NorA pump (Fig. 3b).

Although it did not present a hydrogen bond to the fluorine atom, the interaction observed in these solutions was quite similar to the cryptographic structure of the MATE multidrug efflux pump [41]. In this, the NOR site also presents H-bonds that stabilize their carboxylic acid group through residues Y37, N180 and T202, their ketone radical and a fluorine atom are stabilized by Q34 and its nitrogen-para of the piperazine ring by the oxygen of residue N53 [43].

On the other hand, the best docking pose of $\mathbf{1 3}$ presented an $\mathrm{E}_{\text {bind }}=-6.62 \mathrm{kcal} / \mathrm{mol}$, and it is stabilized by hydrophobic interactions between the quinoline moiety and approximately the same residue side chains I12, I15, F16, I19, and M109. For polar contacts, we observed interactions with residues I15 (3.10 and $3.20 \AA$ ), Q51

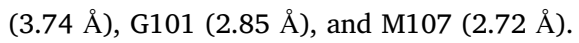

Although these small differences observed in NOR binding sites, the docking studies indicate that both NOR and $\mathbf{1 3}$ recognize the same binding site of the NorA efflux pump, thus implying a probable inhibition by a competitive mechanism as observed in Fig. 2a. Other recent studies using bioinformatic tools report the competitive mechanism between inhibitors and substrates [44]. Previous docking studies of NorA and reserpine show a similar site to that observed in the present study sharing F16, Q51 and R310 as key residues of NorA pump inhibitors [40].

This finding helps to justify the increased activity neither of NOR against $S$. aureus, when associated with guanidine compounds, since the presence of these favors NOR permanence within the bacterial cell, thus allowing it to exert its antibiotic effect.

It is interesting to note that this binding site has a subtle inward projection on the $\mathrm{N}$ - Lobe of NorA. This projection is small enough to hinder the penetration of NOR, however, is adequate to accommodate the guanidine group of the title compounds, being essential to ensure the blockade of NorA pump.

The performed docking showed that the guanidine group of $\mathbf{1 3}$ is projected in the N-Lobe of NorA allowing an H-bond with residue G101, which was considered in this study, as decisive to strengthen the competition between 13 and NOR because it ensures not only greater stability to guanidine compared to NOR, but also helps to minimize the channel hydrophobicity. Additionally, competition between NOR and 13 can be justified by the share of residues I15, F16, I19, Q51, I136, and M107.

Structurally, this projection suggests that guanidine group is important to induce NorA efflux pump inhibition, but the properties and features of the lateral side chain (size, length, the presence of polar 

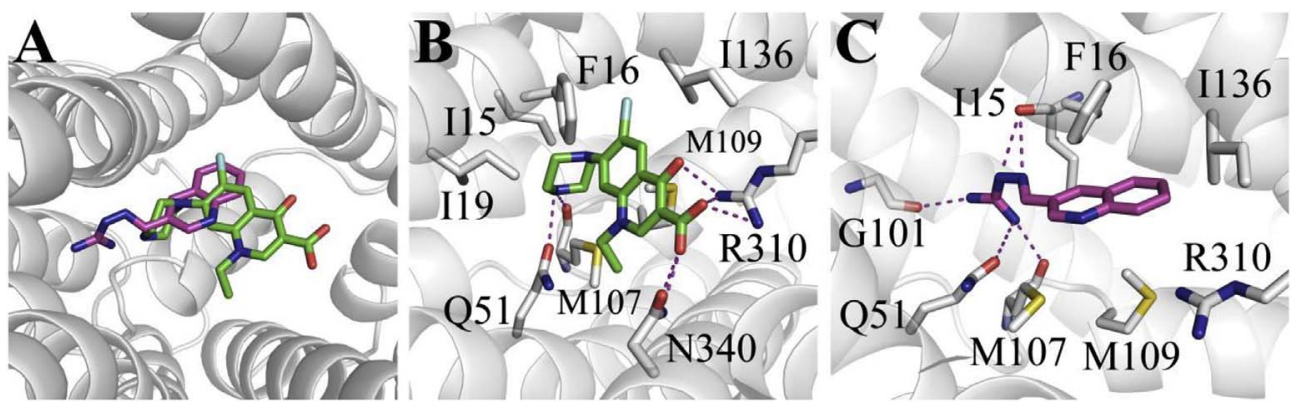

Fig. 3. Representation of NorA/NOR and NorA/ 13 docking. (a) Ribbon representation of both poses shows competition for the same site structure of NOR (green) and $\mathbf{1 3}$ (purple); (b) representation and characterization of NorA/ NOR docking; (c) representation and characterization of NorA/13 docking. Polar contacts presented in purple dotted dashes. Image generated by PyMol software. (For interpretation of the references to color in this figure legend, the reader is referred to the Web version of this article.) groups) of these compounds also seem to have an influence on the blockage.

For example, compounds 10 and 14 with bulky radical groups probably are not well recognized by NorA efflux pump and it may acts by another way as membrane leakage. On the other hand, minor groups as carbonitrile and chlorine present in compounds 2, 3, 12 and 17 (Fig. 1), may act in a similar way increasing the norfloxacin effects equivalent to 13 , as shown in Table 1.

Still, electron donors groups as $-\mathrm{OCH}_{3}$ and $-\mathrm{OH}$ found in compounds 4, 5, 6 and 7 were considered as bad substitutions to NorA blockage, probably due to the hydrophobicity of NorA's site formed mainly by residues I15, F16, M109 and I136 (Fig. 2b and c). The approach of these substituents to the side chains of these residues results in the worst accommodation of these molecules in the active site of NorA pump.

Taken together, NorA efflux pump presents a considerable promiscuity to extrude substrates but it is restricted to medium size molecules with hydrophobic and polar portions as previously proposed [36].

The NorA efflux protein has the as specific substrate ethidium bromide (DNA-intercalating dye) and is the only known resistance mechanism against it $[44,45]$. Therefore, we tested ethidium bromide against the SA-1199B strain to demonstrate that the evaluated AGH derivatives modulate norfloxacin resistance by means of NorA inhibition. On the other hand, perfloxacin, a hydrophobic quinolone, is a poor substrate of the NorA efflux pump so it was used as a negative control $[46,47]$. Thus, the reductions in MIC for ethidium bromide (and not for perfloxacin) (Table 1) reinforce the idea that aminoguanidine hydrazones are putative inhibitors of the NorA efflux system.

Once G's bind to NorA's active site, they prevent antibiotic extrusion, increasing its concentration inside the cell, and consequently its deleterious effects. This was observed 16-fold for norfloxacin and 32fold for ethidium bromide in the in vitro assays (Table 1). It has been detected with other efflux pump inhibitors that act by a competitive mechanism. Such has been proposed; that dipeptide amide (PABN) is itself an RND superfamily competitive substrate. It seems that PABN may recognize and bind to the substrate pocket specific to the potentiated antibiotics. Alternatively, due to a proximal binding site, the EPI may also generate steric hindrance, impairing the antibiotic binding at its affinity site. PA $\beta N$ has been validated against the AcrAB-TolC in a variety of Gram-negative pathogens (K. pneumoniae, E. coli, S. typhimurium, and E. aerogenes) [48].

\section{Conclusion}

In this work, we observed for the first time that AGHs are putative inhibitors of the NorA efflux pump, and can be associated with norfloxacin for the treatment of infections caused by $S$. aureus strains NOR resistant and which overexpress genes for NorA efflux pumps. The reductions in MIC for ethidium bromide up to 32-fold (and not for perfloxacin) reinforce the idea that AGHs are putative inhibitors of the NorA efflux system.

The molecular modeling and docking studies provide support for these in vitro observations, and proved to be a good tool to elucidate the interactions between AGHs and the generated three-dimensional NorA model. The molecular docking involving NorA inhibition showed that AGHs binding on the same norfloxacin binding site and that the guanidine group, seems to affect the NorA efflux pump inhibition. More indepth analysis of the activities of these compounds are necessary since there is no general mechanism for pump inhibition.

However, in our study we observed a potential mechanism of competitive inhibition which allows explaining the restoration of the sensitivity of $S$. aureus cells (SA1199B strain) to norfloxacin and to ethidium bromide.

The results of this study using AGH derivatives as modulators of antibiotic activity is unheard of in this area, are important towards developing focused adjuvants for antibiotic drug therapies against bacterial multidrug resistance.

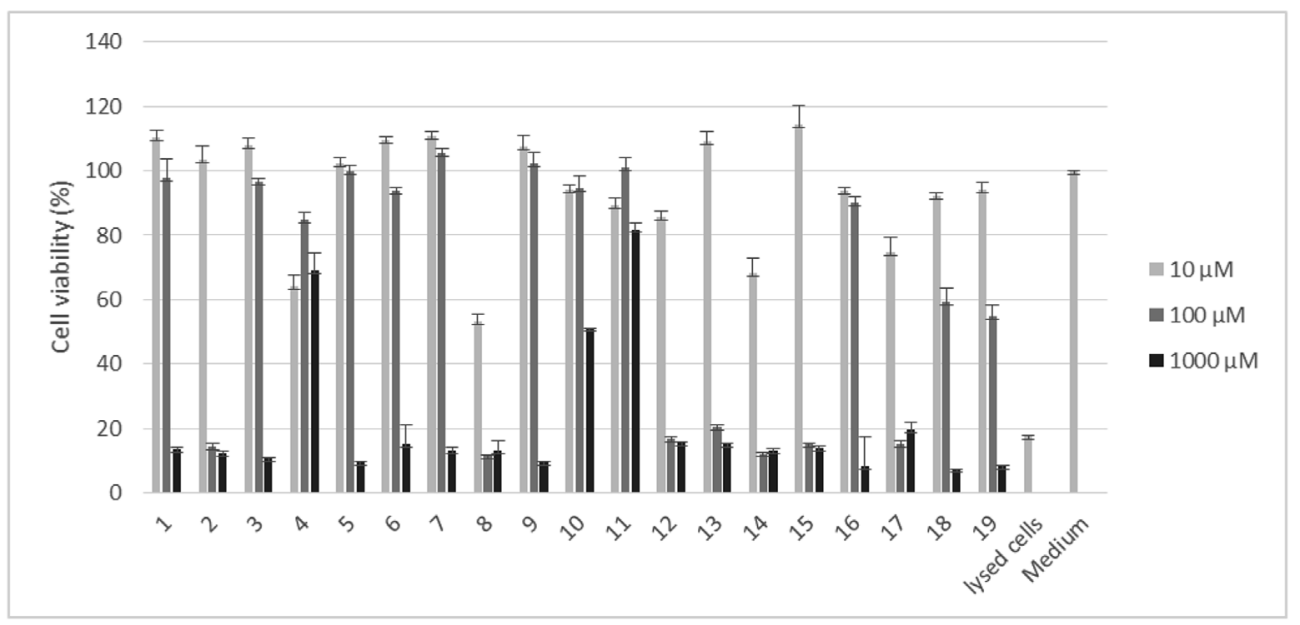

Graph 1. In vitro cytotoxicity activity of AGH derivatives (1-19), at three different concentrations $(10,100$ and $1000 \mu \mathrm{g} / \mathrm{mL})$ in macrophages cell line J774, measured by MTT assay. The bars represent the mean \pm standard deviation of four experiments. 


\section{Acknowledgements}

Authors would like to acknowledge the Brazilian National Council for Scientific and Technological Development (CNPq) for scholarships and for financial support (Grant numbers 305867/2011-3, 478486/ 2011-1), PROPESQ/UEPB and FAPEAL.

\section{Transparency document}

Transparency document related to this article can be found online at http://dx.doi.org/10.1016/j.cbi.2017.12.009.

\section{References}

[1] B. Spellberg, R. Guidos, D. Gilbert, J. Bradley, H.W. Boucher, W.M. Scheld, J.G. Bartlett, J.J. Edwards, The epidemic of antibiotic-resistant infections: a call to action for the medical community from the Infectious Diseases Society of America, Clin. Infect. Dis. 46 (2008) 155-164.

[2] J. Finks, E. Wells, T.L. Dyke, N. Husain, L. Plizga, R. Heddurshetti, M. Wilkins, J. Rudrik, J. Hageman, J. Patel, C. Miller, Vancomycin-resistant Staphylococcus aureus, Michigan, USA, 2007, Emerg. Infect. Dis. 15 (2009) 943-945.

[3] S. Tsiodras, H.S. Gold, G. Sakoulas, G.M. Eliopoulos, C. Wennersten, L. Venkataraman, R.C. Moellering, M.J. Ferraro, Linezolid resistance in a clinical isolate of Staphylococcus aureus, Lancet 358 (2001) 207-208.

[4] G.W. Kaatz, S.M. Seo, Inducible NorA-mediated multidrug resistance in Staphylococcus aureus, Antimicrob. Agents Chemother. 39 (1995) 2650-2655.

[5] E. Smith, E. Williamson, N. Wareham, G.W. Kaatz, S. Gibbons, Antibacterial and modulators of bacterial resistance from the immature cones of Chamaecyparis lawsoniana, Phytochem 68 (2007) 210-217.

[6] A.A. Neyfakh, C.M. Borsch, G.W. Kaatz, Fluoroquinolone resistance protein NorA of Staphylococcus aureus is a multidrug efflux transporter, Antimicrob. Agents Chemother. 37 (1993) 128-129.

[7] A.A. Neyfakh, The mystery of multidrug transporters: the answer can be simple, Mol. Microbiol. 44 (2002) 1123-1130.

[8] A.S. Lynch, Efflux systems in bacterial pathogens: an opportunity for therapeutic intervention? An industry view, Biochem. Pharmacol. 71 (2006) 949-956.

[9] N. Totha, M.V. Reddy, A. Kumar, I.A. Khan, P.L. Sangwan, N.P. Kalia, J.P. Koul, S. Koul, Substituted dihydronaphthalenes as efflux pump inhibitors of Staphylococcus aureus, Eur. J. Med. Chem. 45 (2010) 3607-3616.

[10] B.A. Mitchell, M.H. Brown, R.A. Skurray, QacA multidrug efflux pump from Staphylococcus aureus: comparative analysis of resistance to diamidines, biguanidines, and aminoguanidine hydrazones, Antimicrob. Agents Chemother. 42 (1998) 475-477.

[11] W.O. Foye, B. Almassian, M.S. Eisenberg, T.J. Maher, Synthesis and biological activity of aminoguanidine hydrazones of 2-pyridine and 4-pyridine and 4-quinoline carboxaldehydes, J. Pharm. Sci. 79 (1990) 527-530.

[12] B.B. Hoffman, Catecolaminas, fármacos simpático miméticos e antagonistas dos receptores adrenérgicos, p 163-203, in: A.G. Gilman, J.G. Hardman, L.E. Limbird (Eds.), Goodman \& Gilman: As bases farmacológicas da terapêutica, 10ed, 2003 Rio de Janeiro, RJ, Brazil.

[13] S.D. Larsen, A.M. Connell, M.M. Cudahy, B.R. Evans, P.D. May, M.D. Meglasson, T.J. O'Sullivan, H.J. Schostarez, J.C. Sih, F.C. Stevens, S.P. Tanis, C.M. Tegley, J.Á. Tucker, V.A. Vaillancourt, T.J. Vidmar, W. Watt, J.H. Yu, Synthesis and biological activity of analogues of the antidiabetic/antiobesity agent 3-guanidinopropionic acid: discovery of a novel aminoguanidino acetic acid antidiabetic agent, J. Med. Chem. 44 (2001) 1217-1230.

[14] A. Andreani, A. Leoni, A. Locatelli, R. Morigi, M. Rambaldi, M. Recanatini, V. Garaliene, Potential antitumor agents. Part 29: synthesis and potential coanthracyclinic activity of imidazo $[2,1-b]$ thiazole aminoguanidine hydrazones, Bioorg. Med. Chem. 8 (2000) 2359-2366.

[15] Z. BrzozowskI, F. Saczewski, J. Slawinski, Synthesis of novel 3-amino-2-(4-chloro-2mercaptobenzenesulfonyl)-guanidine derivatives as potential antitumor agents, Eur. J. Med. Chem. 42 (2007) 1218-1225.

[16] R. Bairwa, M. Kakwani, N.R. Tawari, J. Lalchandani, M.K. Ray, M.G.R. Rajan, M.S. Degani, Novel molecular hybrids of cinnamic acids and aminoguanidine hydrazones as potential antitubercular agents, Bioorg. Med. Chem. Lett. 20 (2010) 1623-1625.

[17] M.N. Borges, J.D. Figueroa-Villar, NMR interaction studies of aromatic aminoguanidine hydrazones with micelles: model for mechanism of action of cationic antibiotics, Biopolymers 62 (2001) 9-14.

[18] C.S. Oliveira, B.F. Lira, V.S. Falcão-Silva, J.P. Siqueira-Junior, J.M. Barbosa-Filho, P.F. Athayde-Filho, Synthesis, molecular properties prediction, and anti-staphylococcal activity of N-Acylhydrazones and new 1, 3, 4-oxadiazole derivatives, Molecules 17 (2012) 5095-5107.

[19] N. Ahmed, K.G. Brahmbhatt, S.I. Khan, M. Jacob, B.L. Tekwani, S. Sabde, B. Mitra, I.P. Singh, A.I. Khan, K.K. Bhutani, Synthesis and biological evaluation of tricyclic guanidine analogues of batzelladine $\mathrm{K}$ for antimalarial, antileishmanial, antibacterial, antifungal, and anti-HIV activities, Chem. Biol. Drug. Des. 81 (2013) 491-498.

[20] W.A. Brito, M.G. Dantas, F.H.A. Nogueira, E.F. Silva-Júnior, J.X. Araújo-Júnior T.M. Aquino, E.A.N. Ribeiro, L.G.S. Solon, C.F.S. Aragão, A.P.B. Gomes, Development and validation of HPLC-DAD and UHPLC-DAD methods for the simultaneous determination of guanilhydrazones derivatives employing a factorial design, Molecules 22 (2017) 1394-1409.

[21] P.H.B. França, E.F. Silva-Júnior, P.G.V. Aquino, A.E.G. Santana, J.N.S. Ferro, E.O. Barreto, C.Ó. Pessoa, A.S. Meira, T.M. Aquino, M.S. Alexandre-Moreira, M. Schmitt, J.X. Araújo-Júnior, Preliminary in vitro evaluation of the anti-proliferative activity of guanylhydrazone derivatives, Acta Pharm. Croat. 66 (2016) $129-137$.

[22] V.S. Falcão-Silva, D.A. Silva, M.S.V. Souza, J.P. Siqueira-Junior, Modulation of drug resistance in Staphylococcus aureus by a kaempferol glycoside from Herissantia tiubae (malvaceae), Phytother. Res. 23 (2009) 1367-1370.

[23] NCCLS - National Committee for Clinical Laboratory Standards, Performance Standards for Antimicrobial Susceptibility Testing; Fifteenth Informational Supplement, Clinical and Laboratory Standards Institute, Wayne, PA, USA, 2005 CLSI/NCCLS document M100-S15.

[24] M. Stavri, L.J.V. Piddock, S. Gibbons, Bacterial efflux pump inhibitors from natural sources, J. Antimicrob. Chemother. 59 (2007) 1247-1260.

[25] J.R. de Oliveira, D. de Jesus, L.W. Figueira, F.E. de Oliveira, C. Pacheco Soares, S.E. Camargo, A.O. Jorge, L.D. de Oliveira, Biological activities of Rosmarinus officinalis L. (rosemary) extract as analyzed in microorganisms and cells, Exp. Biol. Med. 242 (2017) 625-634.

[26] M. Biasini, B. Bienert, A. Waterhouse, K. Arnold, G. Studer, T. Schmidt, F. Kiefer, T.G. Cassarino, M. Bertoni, L. Bordoli, T. Schwede, SWISS-MODEL: modelling protein tertiary and quaternary structure using evolutionary information, Nucleic Acids Res. 42 (2014) W252-W258.

[27] D. Jiang, Y. Zhao, X. Wang, J. Fan, J. Heng, X. Liu, W. Feng, X. Kang, B. Huang, J. Liu, X.C. Zhang, Structure of the YajR transporter suggests a transport mechanism based on the conserved motif A, Proc. Natl. Acad. Sci. U. S. A. 110 (2013) 14664-14669.

[28] N. Guex, M.C. Peitsch, SWISS-MODEL and the Swiss-PdbViewer: an environment for comparative protein modeling, Electrophoresis 18 (1997) 2714-2723.

[29] G.M. Morris, R. Huey, W. Lindstrom, M.F. Sanner, R.K. Belew, D.S. Goodsell, A.J. Olson, Autodock4 and AutoDockTools4: automated docking with selective receptor flexibility, J. Comput. Chem. 16 (2009) 2785-2791.

[30] A.W. Schüttelkopf, D.M.F. van Aalten, PRODRG - a tool for high-throughput crystallography of protein-ligand complexes, Acta Crystallogr. D60 (2004) 1355-1363.

[31] W.L. Delano, The PyMOL Molecular Graphics System, DeLano Scientific, San Carlos, CA, USA, 2002.

[32] G. Appendino, S. Gibbons, A. Giana, A.G. Pagani, G. Grassi, M.E. Stavri, E. Smith, M.M. Rahman, Antibacterial cannabinoids from Cannabis sativa: a structure activity study, J. Nat. Prod. 71 (2008) 1427-1430.

[33] A. Coqueiro, L.O. Regasini, P. Stapleton, V.S. Bolzani, S. Gibbons, In vitro antibacterial activity of prenylated guanidine alkaloids from pterogyne nitens and synthetic analogues, J. Nat. Prod. 77 (2014) 1972-1975.

[34] P.L. Sangwan, J.L. Koul, S. Koul, M.V. Reddy, N. Thota, I.A. Khan, A. Kumar, N.P. Kalia, G.N. Qazi, Piperine analogs as potent Staphylococcus aureus NorA efflux pump inhibitors, Bioorg. Med. Chem. 16 (2008) 9847-9857.

[35] C. Vidaillac, J. Guillon, C. Arpin, I. Forfar-Bares, B.B. Ba, J. Grellet, S. Moreau, D.H. Caignard, C. Jarry, C. Quentin, Synthesis of omeprazole analogues and evaluation of these as potential inhibitors of the multidrug efflux pump NorA of Staphylococcus aureus, Antimicrob. Agents Chemother. 51 (2007) 831-838.

[36] J.P. Brincat, E. Carosati, S. Sabatini, G. Manfroni, A. Fravolini, J.L. Raygada, D. Patel, G.W. Kaatz, G. Cruciani, Discovery of novel inhibitors of the NorA multidrug transporter of Staphylococcus aureus, J. Med. Chem. 54 (2011) 354-365.

[37] A.O. Santos-Filho, J.D. Figueroa-Villar, M.T. Araújo, Molecular modeling of the interaction of tryponocide aminoguanidine hydrazones with B-DNA, Bioorg. Med. Chem. Lett. 17 (1997) 1797-1802.

[38] B. Liu, E. Wilson, The antimicrobial activity of CCL28 is dependent on C-terminal positively charged amino acids, Eur. J. Immunol. 40 (2010) 186-196.

[39] C.L. Hannon, E.V. Anslyn, The guanidinium group: its biological role and synthetic analogs, Bioorg. Chem. Front. 3 (1993) 193-255.

[40] F. van Bambeke, J.M. Pages, V.J. Lee, Inhibitors of bacterial efflux pumps as adjuvants in antibacterial therapy and diagnostic tools for detection of resistance by efflux, Front. Anti-Infect. Drug Disc. 1 (2010) 138-175.

[41] N.P. Kalia, P. Mahajan, A. Mehra-Nargotra, J.P. Sharma, I.A. Koul-Khan, Capsaicin, a novel inhibitor of the NorA efflux pump, reduces the intracellular invasion of Staphylococcus aureus, J. Antimicrob. Chemother. 67 (2012) 2401-2408.

[42] P. Benkert, M. Biasini, T. Schwede, Toward the estimation of the absolute quality of individual protein structure models, Bioinformatics 27 (2011) 343-350.

[43] Y. Tanaka, C.J. Hipolito, A.D. Maturana, K. Ito, T. Kuroda, T. Higuchi, T. Katoh, H.E. Kato, M. Hattori, K. Kumazaki, T. Tsukazaki, R. Ishitani, H. Suga, O. Nureki, Structural basis for the drug extrusion mechanism by a MATE multidrug transporter, Nature 496 (2013) 247-251.

[44] N.R. Guz, F.R. Stermitz, J.B. Johnson, T.D. Beeson, S. Willen, Flavono lignan and flavone inhibitors of a Staphylococcus aureus multidrug resistance pump: structureactivity relationships, J. Med. Chem. 44 (2001) 261-268.

[45] G.P. Tegos, M. Haynes, J.J. Strouse, M.M. Khan, C.G. Bologa, T.I. Oprea, L.A. Sklar, Microbial efflux inhibition; tactics \& strategies, Curr. Pharm. Des. 17 (2011) 1291-1302.

[46] F.R. Stermitz, L.N. Scriven, G. Tegos, K. Lewis, Two flavonols from Artemisa annua, which potentiate the activity of berberine and against a norfloxacin resistant strain of Staphylococcus aureus, Planta Med. 68 (2002) 1140-1141.

[47] B. Marquez, Bacterial efflux systems and efflux pumps inhibitors, Biochim 87 (2005) 1137-1147.

[48] C. Kourtesi, A.R. Ball, Y.Y. Huang, S.M. Jachak, D.M.A. Vera, P. Khondkar, S. Gibbons, M.R. Hamblin, G.P. Tegos, Microbial efflux systems and inhibitors: approaches to drug discovery and the challenge of clinical implementation, Open Microbiol. J 7 (2013) 34-52. 